Methylphenidate and other drugs used to treat $\mathrm{ADHD}$ are likely to produce similar benefits. ADHD is common in cerebral palsy, particularly in diplegia associated with prematurity, but rarely diagnosed. Increased awareness and treatment of comorbid ADHD may make the difference between achieving and maintaining walking or becoming wheelchair dependent, with important implications for quality of life and life expectancy.

\section{G112(P) EARLY PUBERTAL CHANGES IN NEURODISABLED CHILDREN - AN UNDER DIAGNOSED ISSUE?}

doi:10.1136/archdischild-2013-304107.124

R Mithyantha, Z Bassi. Paediatric Neurodisability Department, Alder Hey Childrens' Hospital, Liverpool, UK

Aim Evaluation of pubertal changes is an easily missed component in the assessment of the complex neurodisabled child.There is limited literature available in the UK regarding pubertal changes in these children. Available evidence suggests an earlier age of commencement of puberty and an increased incidence of precocious puberty in comparison with the general population. We aim to identify the characteristics of early pubertal changes amongst patients seen within a tertiary neurodisability setting.

Methods Retrospective data was collated from patients with neurodisability and premature pubertal changes who were seen over an 18 month period. Patients were evaluated through clinical examination and subsequently referred for endocrine assessment. Hormonal assays and bone age estimation were conducted where appropriate. Results 13 children (male: female $=2: 11$ ) with neurodisability and early pubertal changes were identified. All 13 children had learning difficulties with the majority(84\%) placed in special schools. $\mathrm{CP}(38 \%)$ and SLD of unknown origin(38\%) were the most common underlying diagnosis. Age at presentation was 3-8.5 years (median age: boys $=5.75$ years, girls $=6$ years $)$. Of the 11 girls, $54.5 \%(n=6$, median age: 4.7 years)were diagnosed with central precocious puberty and presented with thelarche and pubarche (tanner stage 2). They had advanced bone age and hormonal levels in pubertal range. 1 girl was diagnosed with idiopathic precocious puberty . The remaining children, 2 boys(mean age $5.75 y$ ears) and 3 girls (mean age 6.8years) presented with isolated thelarche or pubarche , had prepubetral sex hormone levels, normal or marginally increased bone age, normal or high adrenal steroid levels. These children were diagnosed with premature or exaggerated adrenarche and did not warrant treatment. Results of investigations are pending in 1 child. $6 / 7$ children diagnosed with precocious puberty were treated with GNRH analogues (parents refused treatment for 1 child)

Conclusions In our review, true precocious puberty was identified in younger children as compared to older children who presented with isolated secondary sexual characteristics. A significant proportion of these children required hormonal treatment. Thus, assessment of pubertal changes should be an essential component of the multipronged evaluation of children with neurodisability.

\section{G113(P) A SURVEY OF CARERS' SATISFACTION WITH GASTROSTOMY TUBE FEEDING FROM A TERTIARY CHILDREN'S HOSPITAL}

doi:10.1136/archdischild-2013-304107.125

MO Ogundele, Z Bassi. Community Paediatrics, Royal Children's Foundation NHS Hospital Alder Hey, Liverpool, UK

Gastrostomy tube (GT) feeding is a well recognised solution for preventing growth failure and enhancing quality of life for the children with neurologic impairment and their caregivers.

Aims To assess the level of parents/carers satisfaction and experience after gastrostomy tube insertion among children with moderate to severe learning and physical disabilities.
Method 50 parents/carers of children attending special education schools for moderate to severe learning and physical disabilities in a local district were requested to participate in a survey by completing a 23 -item questionnaire. A total of 15 respondents (so far) were analysed.

Results The age of the children ranged from 3 to 18 years (Median 10.8 years) with a M:F sex ration of 1:2. Gastrostomy was performed between 5 and 204 months of age (Median of 4.6 years). $40 \%$ of the carers wished they had the GT inserted earlier. The commonest problems ever associated with feeding were vomiting/regurgitation (80\%), poor growth/weight gain (73\%), coughing/choking and recurrent chest infections (66\% each). The main problem for each child was reported to be improved in $80 \%$ and remained unchanged in $13 \%$. The frequency of feeding either remained the same or increased (36\% each) in most children while the duration of each feed either reduced (70\%) or remained the same.

More than $50 \%$ of the carers reported positive effect of GT feeding in relation to ease of medication administration, the Child's Quality of life, happiness and ease of handling, improved growth/weight gain, reduced vomiting, increased time available for minding other children, better control of pain, epilepsy and chest infections (Table 1).

\section{Abstract G113(P) Table 1}

\begin{tabular}{|l|c|c|c|c|}
\hline \multicolumn{1}{|c|}{ Effect of GTF } & $\begin{array}{c}\text { Total } \\
\text { Respondents }\end{array}$ & $\begin{array}{c}\text { \% } \\
\text { Improved }\end{array}$ & \% Same & \% Worse \\
\hline Ease of Medications & 15 & 93.3 & 6.7 & 0.0 \\
\hline QoL_for Child & 14 & 92.9 & 6.7 & 0.0 \\
\hline Main Problem before GTF & 15 & 80.0 & 13.3 & 1.1 \\
\hline Vomiting/Regurgitation & 10 & 80.0 & 13.3 & 0.0 \\
\hline Poor Growth/Wt Gain & 12 & 75.0 & 13.3 & 1.1 \\
\hline Time_for Other Children & 15 & 66.7 & 20.0 & 0.0 \\
\hline Happier_Child & 14 & 64.3 & 26.7 & 0.0 \\
\hline Child Easier to Handle & 14 & 57.1 & 20.0 & 3.2 \\
\hline Pain & 7 & 57.1 & 20.0 & 0.0 \\
\hline Epilepsy & 7 & 57.1 & 20.0 & 0.0 \\
\hline Recurrent Chest_Infections & 9 & 55.6 & 26.7 & 0.0 \\
\hline QoL_for Carer & 14 & 50.0 & 40.0 & 1.1 \\
\hline GP_Consultations & 14 & 50.0 & 46.7 & 0.0 \\
\hline Time_for Self (Carers) & 15 & 46.7 & 26.7 & 4.3 \\
\hline Ease Finding Minders & 14 & 28.6 & 33.3 & 5.4 \\
\hline Stiffness/Dystonia & 6 & 16.7 & 26.7 & 1.1 \\
\hline Carers Strained_Relationships & 11 & 9.1 & 26.7 & 1.1 \\
\hline Ease of Back_to Work & 13 & 7.7 & 6.7 & 0.0 \\
\hline
\end{tabular}

(ey: QoL = Quality of life

Conclusion Over two thirds of the carers expressed overall improvement of their children's feeding problems with the gastrostomy feeding, helping to resolve common problems such as vomiting/regurgitation, poor growth/weight gain, coughing/choking and recurrent chest infections. The healthcare professionals need to provide advice and necessary information to help the carers make the decision at an early stage.

\section{G114(P) CHILDREN WITH EXCEPTIONAL HEALTHCARE NEEDS: "PERSONHOOD" AND MORAL STANDING IN COMMUNITY}

doi:10.1136/archdischild-2013-304107.126

JM Balfe. Community Child Health, Tallaght Hospital, Dublin, Ireland

For almost 400 years philosophers have debated the attributes necessary for "personhood" or full moral status. While some argue that defining the moral status of children with profound intellectual disability and exceptional healthcare needs is an academic pursuit which fails to ensure that their best interests are met, I believe that the issue warrants careful consideration. Personhood confers moral status and protection by moral norms, if children with exceptional healthcare needs do not have full moral status, it could be argued that they are not holders of human rights.

A number of criteria necessary for "Personhood" have been proposed including attributes of the individual, either biological or 
psychological, or factors regarding an individual's membership of a species or community and their potential to develop relationships within that group. Although children with profound intellectual disability do not have the necessary cognitive ability to meet the psychological criteria for personhood, I argue that as members of the same species all human have moral status and deserve protection.

By considering children and adults with profound intellectual disability as holders of full moral status, an obligation to protect their rights is conferred. Society has an obligation to ensure that their best interests are met. The law has repeatedly stated that competent adults have a right to refuse treatment; the right to die is inherent in law. In this respect if children with exceptional health care needs have full moral status they do not only have the right to medical treatment but also the right to be protected from unnecessary, futile and potentially painful procedures in the absence of any hope of cure. Thus when considering the best interests of children with exceptional healthcare needs, attempts must be made to ensure that their rights as persons are respected.

\section{International Child Health/British Association of General Paediatrics}

\section{G115 REHABILITATION OF CHILDREN WITH NEURODISABILITY FOLLOWING BRAIN INJURY IN MALAWI: PERSPECTIVES OF FAMILIES AND HEALTH-WORKERS}

doi:10.1136/archdischild-2013-304107.127

${ }^{1} \mathrm{~A}$ Holme, ${ }^{2} \mathrm{M}$ Mallewa, ${ }^{2} \mathrm{D}$ Chinguo, ${ }^{3} \mathrm{C}$ Mahebere-Chirambo, ${ }^{4} \mathrm{MJ}$ Gladstone. ${ }^{1} \mathrm{Centre}$ for Child \& Adolescent Health, School of Social \& Community Medicine, University of Bristol, Bristol, UK; ${ }^{2}$ Queen Elizabeth Central Hospital, College of Medicine, Blantyre, Malawi; ${ }^{3}$ Malawi Liverpool Wellcome Trust, Blantyre, Malawi; ${ }^{4}$ Women's \& Children's Health, Institute of Translational Medicine, University of Liverpool, Liverpool, UK

Aims Rehabilitation services in Malawi are limited despite the burden of paediatric neurodisability secondary to conditions such as cerebral malaria, meningitis, encephalitis and traumatic brain injury. This hospital-based study aims to explore the perceptions and experiences of parent/carers and health-workers caring for children with neurodisability following brain injury, in order to inform the development of a capacity-building programme to improve rehabilitation services.

Methods The study was conducted in the Paediatrics department of an urban tertiary hospital in Malawi and utilised qualitative methodology. Sampling was purposive with recruitment via Paediatrics staff. Fourteen interviews were completed with parents/carers of children affected by recent brain injuries, and ten interviews and four focus-groups with health-workers. Analysis involved a thematic framework approach.

\section{Results}

Health-workers With limited resources, management of acute conditions is prioritised over rehabilitation. Lack of confidence, knowledge, and a sense of 'there's nothing we can do' hampers health-workers' rehabilitation efforts, despite many suggesting that simple things make a difference. Bias exists towards managing physical disability rather than impairments of cognition, speech or behaviour. Concerns were raised about the wider impact of neurodisability on the family, including the risk of child abuse and neglect. Counselling, including information giving, was identified as a priority. Health-workers recognised that effective rehabilitation could not be achieved unless parents/carers first understood and accepted their child's condition.

Parents/Carers Reports of poor communication between healthworkers and parents/carers were common. Parents often had not understood or been adequately informed about investigations, diagnosis or management of their child's condition. This contributed to unrealistic expectations about prognosis, misunderstanding about underlying causes, and lack of continued rehabilitation in the community. The burden of caring for a child with neurodisability, and the financial and opportunity costs this entails was strongly evident. Main issues were lack of mobility in their child, feeding, continence and speech difficulties. Religion was a key source of support.

Conclusion Limited resources, lack of health-worker training, bias towards physical disability, and poor communication, are key factors inhibiting rehabilitation of children with brain injury in a hospital-setting in Malawi. A programme to improve rehabilitation services should address these issues as priority.

\section{G116 PROFILE OF DENGUE VIRAL INFECTION AMONG CHILDREN TREATED IN A COMMUNITY HOSPITAL OVER ONE YEAR AND COMPARISON OF EFFICACY OF NS1 ANTIGEN ASSAY WITH MAC-ELISA FOR DIAGNOSIS OF DENGUE}

doi:10.1136/archdischild-2013-304107.128

S Padankatti, D Elangovan, K Thomas. Department of Pediatrics, Sundaram Medical Foundation, Dr Rangarajan Memorial Hospital, Chennai, India

Aims Early diagnosis of Dengue is important to improve patient outcomes and promote timely public health interventions. This study aimed at

1. studying the profile of paediatric Dengue viral infection,

2. comparing efficacy of NS1 antigen assay with MAC-ELISA for diagnosis of dengue, during both acute and convalescent phases and

3. assessing time frame for positivity of these tests.

Methods This was a prospective cohort study in the Paediatric department of a community hospital in a tropical developing country, during the period July 2011 to July 2012. The Institutional Review Board approved the study. Written informed consent was obtained from all parents. Clinical features of 178 children who presented with Dengue infection were studied. Dengue NS1 antigen, IgM and IgG ELISA were done on the day of admission irrespective of duration of fever. Other laboratory tests were done as per current protocol. Chi-square test and proportion test were used for analysis.

Results Majority of children (37.7\%) was in the age group 11 to 15 years. $59.4 \%$ were males. The proportion of males was higher in extremes of age groups. Common clinical manifestations were fever (91.6\%), rash (35.8\%), hepatomegaly (33\%), ascites $(11.3 \%)$, bleeding manifestations (17\%) and pleural effusion (3.7\%). $21.7 \%$ had Dengue Haemorrhagic Fever and 7.5\% had Dengue Shock Syndrome. Laboratory findings were thrombocytopenia (77\%), leucopenia $(24.5 \%)$ and raised SGPT $(63.2 \%)$.

$33 \%$ of children presented in acute phase ( $\leq 4$ days) and $67 \%$ in convalescent phase (5-7 days: $53.8 \%,>7$ days: $13.2 \%$ ).

$94.3 \%$ were positive either for NS1 Ag or IgM antibody or both and $5.7 \%$ were clinically treated as dengue fever.

NS1 and IgM ELISA positivity were $88.6 \%$ and $51.4 \%$ respectively in acute phase and $63.38 \%$ and $92.8 \%$ respectively in convalescent phase $(p<0.001)$. Time frame for positivity of these tests is shown in table 1. NS1 antigen detection was very good in acute phase of both primary and secondary infections, but low in convalescent phase of secondary infections.

Conclusions Early and accurate diagnosis of dengue infection can be made with typical clinical features and laboratory investigations. NS1 Antigen in the acute phase and IgM ELISA in the convalescent phase are good diagnostic tools. 